www. revist a d y o.com

\title{
Marco de evaluación de los sistemas de información de una organización manufacturera
}

\author{
Puche-Regaliza JC, Costas-Gual J, de la Fuente-García D, Puente-García J, \\ Fernández-Quesada I
}

Recibido: 12 de Enero de 2021

Aceptado: 21 de Abril de 2021

https://doi.org/10.37610/dyo.v0i74.599

\section{Resumen}

El propósito de este trabajo es desarrollar un marco de evaluación de los sistemas de información en una organización manufacturera siguiendo el Viable System Model y el paradigma Lean Manufacturing. Desarrollamos los puntos clave para tal evaluación examinando las necesidades de los agentes de la organización, la conversión de tales necesidades en requerimientos para disponer de datos, la transformación de datos en información, la toma de decisiones basada en conocimiento, la evaluación del rendimiento y la orientación de los esfuerzos de cambio.

\section{Palabras clave}

Sistemas de Información; Sistemas de Soporte a la Toma de Decisiones; Organizaciones Manufactureras; Viable System Model; Lean Manufacturing.

\section{Introducción}

Las organizaciones en general y las organizaciones manufactureras o dedicadas a la producción en particular, buscan el logro de una serie de objetivos tanto a corto (producir para satisfacer la demanda) como a largo plazo (desarrollar la competitividad) (Porter, 1987; Koontz and Weihrich, 1990; Cuatrecasas, 2000). De entre todos ellos, Goldratt (2010) destaca como principal objetivo de una organización, ganar dinero hoy y mañana.

Para lograr tales objetivos, las organizaciones manufactureras definen los procesos orientados al cliente

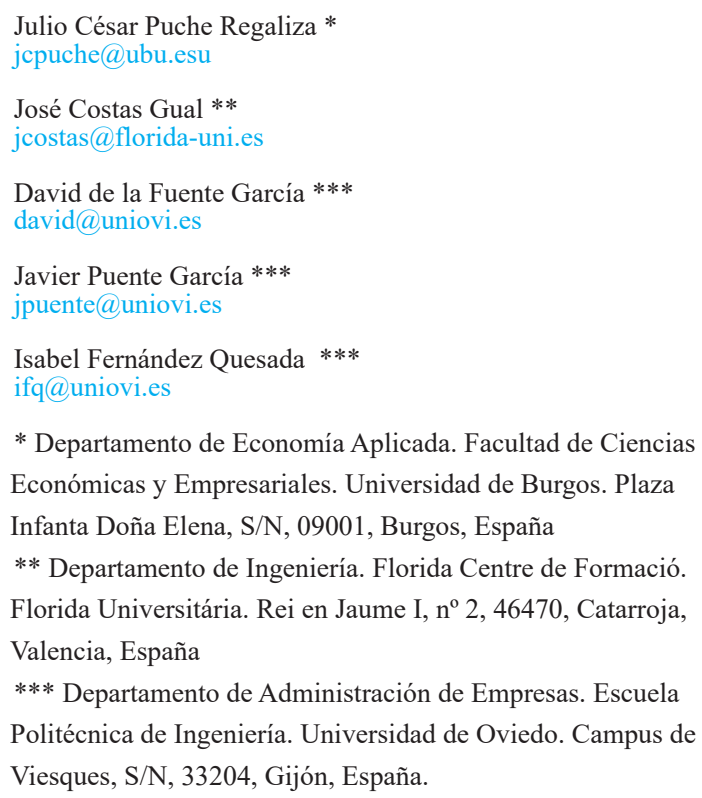

* Departamento de Economía Aplicada. Facultad de Ciencias Económicas y Empresariales. Universidad de Burgos. Plaza Infanta Doña Elena, S/N, 09001, Burgos, España

** Departamento de Ingeniería. Florida Centre de Formació. Florida Universitária. Rei en Jaume I, nº 2, 46470, Catarroja, Valencia, España

*** Departamento de Administración de Empresas. Escuela Politécnica de Ingeniería. Universidad de Oviedo. Campus de Viesques, S/N, 33204, Gijón, España.

(COP) que constituyen su cadena de valor. La cadena de valor requiere un conjunto de procesos orientados al soporte (SOP), tales como procurar planos de piezas, instalar y mantener medios productivos, reclutar y ca-pacitar personas, etc. El sistema de procesos se completa con los procesos orientados al gobierno de la gestión (MOP), como, por ejemplo, el despliegue estratégico, aprobar y gestionar inversiones, gestionar el cambio y la mejora continua, establecer criterios de evaluación del rendimiento con indicadores cuantitativos para medir los resultados, etc. (Frazelle, 2001).

Los sistemas de información desempeñan tres papeles fundamentales en la existencia presente y futura de las organizaciones: (1) facilitan la coreografía de los procesos (flujo de información), (2) proporcionan los registros documentados para trazabilidad y rendición de cuentas, y (3) son el vehículo para la inteligencia sobre el entorno de la organización. Los actores de la organización colaboran para transformar datos en información, usan el conocimiento (talento corporativo) para tomar decisiones soportadas por información, y capturan las lecciones aprendidas por la vía experimental. En consecuencia, el adecuado diseño de los sistemas de información es crítico para asegurar la viabilidad de una organización (Pérez Ríos et al., 2008).

\section{Objetivo}

El propósito de este trabajo es desarrollar un marco de evaluación que permita examinar los sistemas de información, a la par que identifique y priorice diferentes oportunidades de mejora, en una organización manufacturera siguiendo el Viable System Model (VSM) y el paradigma Lean Manufacturing (LM). 
La elección del VSM y del paradigma LM se basa en su superioridad científica frente a otras alternativas ofrecidas comúnmente por la Teoría de Organización y por otros paradigmas productivos de comportamiento respectivamente. Para profundizar en esta justificación puede consultarse Beer $(1979,1981)$ para el primero, y Womack et al., (1990) para el segundo.

\section{Metodología}

A continuación, revisamos los conceptos fundamentales del VSM y del paradigma LM.

\subsection{Viable System Model (VSM)}

La Cibernética Organizacional es una metodología enmarcada en el denominado enfoque sistémico que aplica los principios relacionados con la "comunicación y el control", propios de la Cibernética, a las organizaciones. En este ámbito, destacamos el VSM (Figura 1) que, integrado con otros componentes de la Cibernética Organizacional, establece las condiciones necesarias y suficientes para que una organización sea viable, implicando la existencia de los subsistemas o funciones estructurales denominados por su propio autor, Stafford Beer: Sistema Uno (unidades operacionales), Sistema Dos (coordinación de las unidades operacionales), Sistema Tres (integración y sinergia), Sistema Cuatro (inteligencia y adaptación) y Sistema Cinco (definición de identidad y política). A éstos se añade el Sistema Tres* (auditoría) como complemento del Sistema Tres (Beer, 1985).

Las unidades operacionales son las responsables de producir y entregar al entorno los bienes o servicios que la organización produce. Cada una de ellas representa de manera autónoma, una línea de actividad, producto, proyecto, etc. El resto de los subsistemas, tienen como misión servir a estas unidades operacionales, es decir, contribuir a que cumplan con su propósito.

Dado que pueden presentarse conflictos entre las actividades que desarrollan estas unidades operacionales, es necesario un subsistema de coordinación que permita amortiguar las oscilaciones que se puedan producir entre ellas, generadas por el intento de cada una de lograr sus propios fines. Es decir, incluye los mecanismos necesarios para coordinar las actividades que se realizan en dichas unidades operacionales.

El subsistema de integración y sinergia se ocupa del interior de la organización en tiempo real, intentando mantener su estabilidad interna. Tiene una visión del conjunto de todas las unidades operacionales, siendo por tanto el encargado de identificar potenciales sinergias que pueden aparecer entre ellas, integrando y optimizando el funcionamiento en conjunto de las unidades operacionales. Este subsistema es el responsable de la adecuada asignación de recursos a cada unidad operacional y de monitorizar el funcionamiento de éstas a través de la rendición de cuentas que tienen que realizar. Además, el subsistema de integración y sinergia también tiene que intervenir directamente sobre las unidades operacionales, resolviendo aquellos problemas que el subsistema de coordinación no ha sido capaz de resolver. Para ello, utilizará su autoridad transmitiendo información, instrucciones, directrices, etc. Apoyando al subsistema de integración y sinergia aparece el subsistema de auditoría, encargado de aportar información directamente desde las unidades operacionales, evitando confiar en la información que éstas envían al subsistema de integración y sinergia. Representa mecanismos de control y revisión tanto internos como externos.

El subsistema de integración y sinergia debe gestionar los riesgos intrínsecos de la organización, pero no es capaz de reconocer los potenciales riesgos que pueden aparecer en su entorno. Por lo tanto, requerimos una función estructural que re-suelva este inconveniente. Esta función estructural está representada por el subsistema de inteligencia y adaptación. En el subsistema de inteligencia y adaptación, los cambios del entorno son detectados, priorizados y analizados con referencia a los objetivos principales de la organización, estableciendo espacios de decisión y proyectos de gestión del cambio. Se ocupa por tanto del exterior y del futuro de la organización, con la finalidad de mantener a ésta constantemente preparada para el cambio. El subsistema de inteligencia y adaptación idealmente estará formado por la "sala de operaciones", donde son explorados de forma continua diferentes escenarios futuros para ayudar a la toma de decisiones que incrementen la probabilidad de lograr el futuro deseado.

Como cierre del conjunto, tenemos el subsistema de definición de identidad y política. Esta función estructural es responsable de la formulación de los principios y metas de la organización, desempeñando un importante papel en la preservación de su identidad. Debe asegurar que la organización se adapte al entorno manteniendo al mismo tiempo un grado adecuado de estabilidad interna. Ha de lograr por tanto un adecuado balance entre el subsistema de integración y sinergia (interior y corto plazo) y el subsistema de inteligencia y adaptación (exterior y largo plazo), a veces contradictorios. 
Figura 1 Viable System

Model (Beer, 1985).

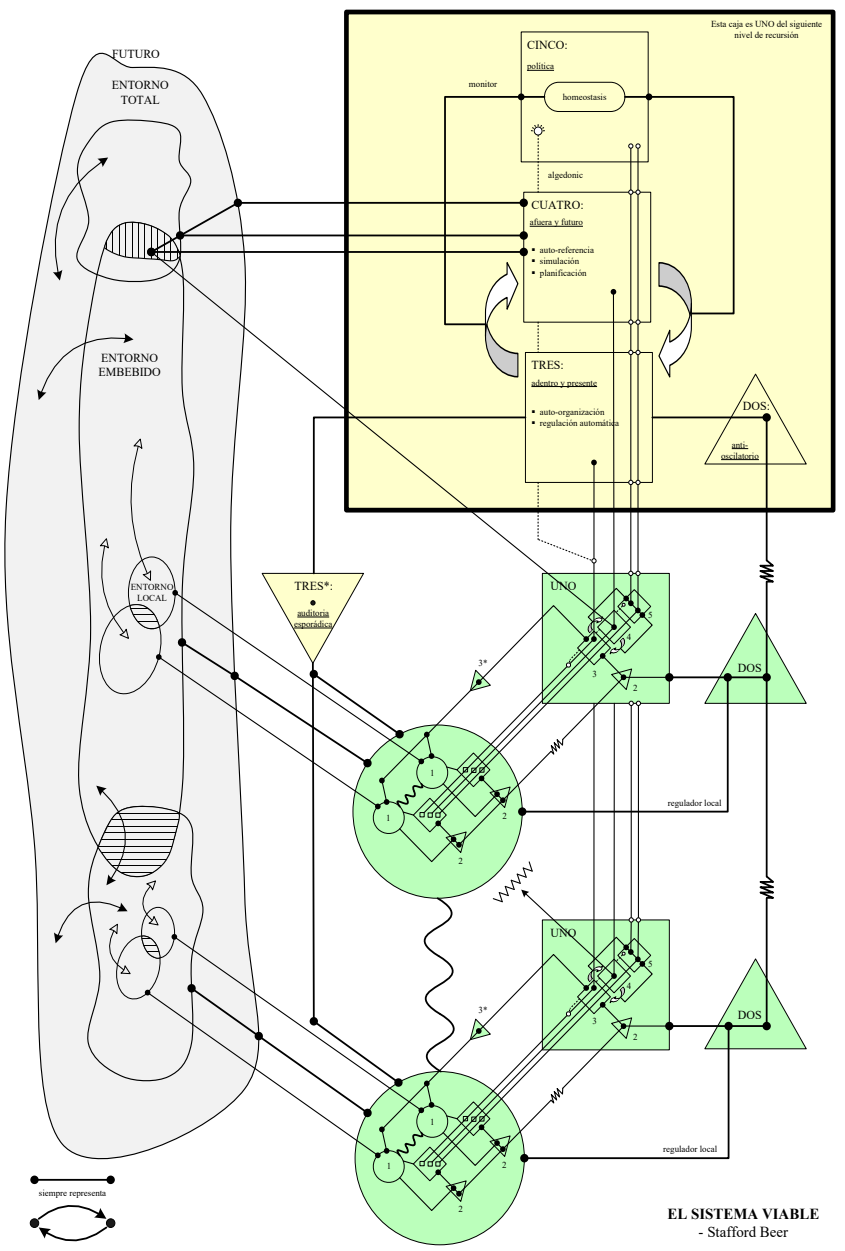

Otro aspecto esencial del VSM es su carácter recursivo. El Principio de Recursión (otro componente de la Cibernética Organizacional) expone que todo sistema viable contiene sistemas viables $\mathrm{y}$, a su vez, forma parte de sistemas que también son viables. En la Figura 1 se puede ver como en cada unidad operacional está contenida una réplica exacta de la estructura del Modelo. La consecuencia directa de esta recursividad es que cualquier sistema viable, independientemente del nivel de recursión que ocupe, debe contener los cinco subsistemas o funciones estructurales definidos por el Modelo, es decir, la viabilidad del sistema requiere que las cinco funciones estructurales existan, de manera recursiva, en todos los niveles de la organización.
Además de los cinco subsistemas o funciones estructurales y el concepto de recursión, el VSM presenta una diversidad de relaciones de comunicación y transmisión que los conectan entre sí y con el entorno de la organización. Estas relaciones persiguen lograr un equilibrio continuo en la interacción entre dichos subsistemas. El equilibrio se logra cuando la información (tanto en cantidad, como en contenido, como en formato) que llega desde un subsistema a otro y viceversa, es la adecuada para que la relación entre ambos sea una relación dinámica pero armónica, en el sentido de que funciona en la forma deseada por las dos partes (Pérez Ríos et al., 2008). 
Figura 2 Elementos de una relación de comunicación y transmisión (Pérez Ríos et al., 2008)

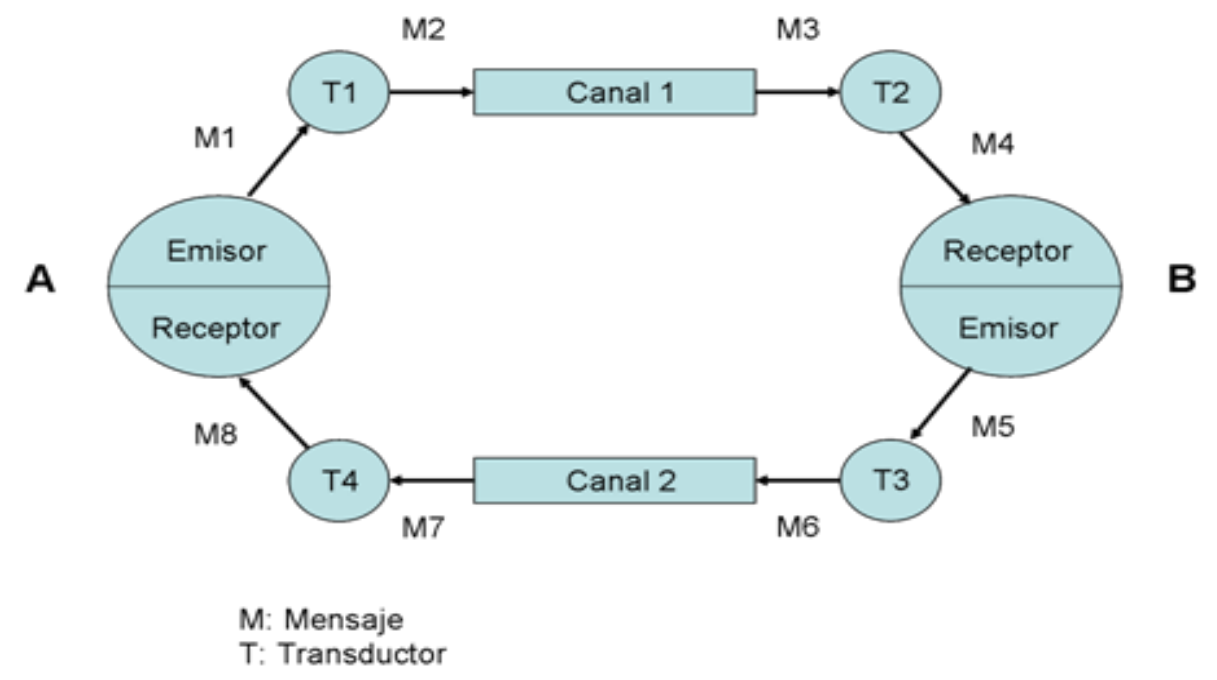

Cada una de las relaciones de comunicación y transmisión entre dos subsistemas de la organización o bien entre un subsistema y el entorno, está compuesta por ocho componentes (Figura 2). El emisor (A), el "transductor" que convierte la información que sale del emisor para que sea inteligible para el receptor, el canal de comunicación que debe ser capaz de conducir la cantidad de información por unidad de tiempo que se le requiere, de nuevo el "transductor" que descodifica la información y la convierte al formato adecuado para que, por último, la interprete el receptor (B). Y en el camino inverso (receptor-emisor) que ahora pasarían a permutar sus papeles (emisorreceptor), volvemos a encontrarnos con los mismos cuatro elementos, pero en el camino inverso (Pérez Ríos et al., 2008).

\subsection{Lean Manufacturing (LM)}

El paradigma LM nace a partir del enfoque sistémico ofrecido por la filosofía Toyota Production System (Figura 3). La característica esencial y diferenciadora respecto a otros paradigmas de fabricación es que en el paradigma
LM el centro de interés está en poner al cliente al mando del sistema productivo. De esta manera, el ideal del paradigma LM es producir al ritmo exacto de la demanda. Cada cliente, con cada orden, está tirando de la cadena de producción, hacia atrás, de forma reiterada, en cada paso de proceso (Womack et al., 1990).

Este paradigma busca la continua reducción del desperdicio de la cadena de valor, bien entendido que el desperdicio es todo aquello que no añade valor para el cliente. La actitud crítica en el juicio sobre el estado en curso de la cadena de valor y el esfuerzo reiterado en la reducción de desperdicio requiere el cultivo de destrezas en solución de problemas. Todo ello conduce a un ambiente de trabajo donde el desafío de nuevos retos es bien recibido y, por lo tanto, a una organización que aprende (abordando los problemas en lugar de esconderlos) y que cada día es más eficaz en satisfacer a los clientes y más eficiente en realizar su actividad (menos desperdicio). Los operarios aprenden, los directivos estimulan el ensayo y la verificación, se incrementa el compromiso con los clientes y las relaciones existentes entre los diferentes componentes de la organización suben su nivel de competencia, agilidad, eficacia y resultados en lugar de operar sólo bajo la premisa de "regulación" (Liker and Meier, 2006). 
Figura 3 Toyota Production

System (Liker, 2004)

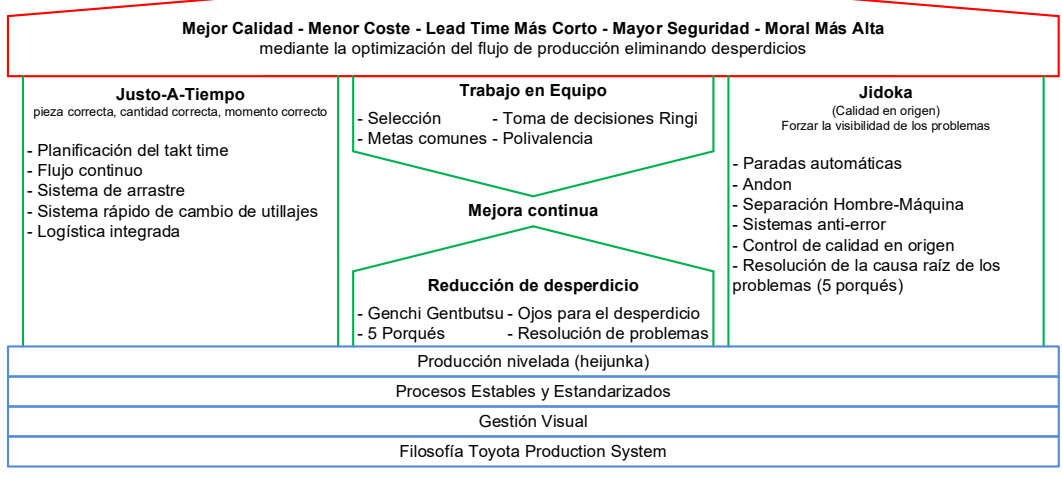

El paradigma LM se apoya en cuatro pilares básicos (Figura 3) que conducen a la estabilidad y como consecuencia a la sostenibilidad de la organización (Liker and Meier, 2006):

- El pilar de la filosofía (conjunto de valores y principios que guían la conducta de los agentes de la organización), extendido a crear valor para los clientes, los empleados, la cadena de suministro y para la comunidad en general.

- El pilar de los procesos, entendido en el sentido de que los resultados (productos o servicios) son consecuencia de la naturaleza de los procesos. Este pilar se despliega en las reglas de flujo, que se opone al almacenamiento; tirar, que sitúa al cliente y la demanda al mando del proceso, y se opone a empujar la producción siguiendo el paradigma de Mass Production (MP): “que la línea no pare"; normas, que enfatiza la aplicación del método científico en la definición cuidadosa y detallada al establecer cómo han de ser las operaciones y los controles para poder detectar y solucionar los problemas sobre la base de la repetitividad del proceso en lugar de que cada problema es diferente cuando el proceso se halla cargado de variedad no requerida (causas asignables) y; mejora, que niega la complacencia, aboga por el desafío continuo, y con ello, a elevar el estándar una y otra vez, frecuentemente, hacia niveles más altos.

- $\quad$ El pilar de las personas, que establece el valor de hacer crecer continuamente a las personas en su dimensión humana, social y técnica.

- El pilar de solución de problemas, que aplicando la regla parar-para-arreglar, crea la cultura de ir en busca de desafíos cada vez más difíciles en vez de huir de los problemas y conformarse con soportar los mismos problemas una y otra vez (como cuando la organización se limita a segregar las piezas defectuosas y enviarlas a una estación de retrabajo). Por lo tanto, un problema es visto como un signo de una patología de la organización actual, y como consecuencia, presenta la oportunidad de diagnosticar las causas raíz del mismo, a fin de transformar la organización hacia otra cuya naturaleza global es superior. Tanto es así, que el proceso de tratamiento de no conformidades es objeto de una definición radicalmente distinta en una organización bajo el paradigma LM, que bajo el paradigma MP. En primer lugar, porque un problema en el paradigma LM recibe una respuesta rápida desde el nivel más bajo de la organización, es decir, desde los grupos autónomos de personas (GAP). Esta respuesta rápida es factible aplicando un protocolo de diagnóstico envasado. Por ejemplo, en fichas de diagnóstico rápido que permiten seguir una secuencia priorizada de descarte de causas directas potenciales de la no conformidad que acaba de ser detectada en la organización gracias al conocimiento de los equipos de análisis modal de fallos, efectos y criticidad (FMEA). Los equipos FMEA estudian el árbol de fallos, que parte de la función proceso, sigue con su despliegue en subfunciones, asocia los elementos de trabajo implicados en cada subfunción y finalmente, deriva los riesgos (lo que puede ir mal en alguno de los elementos de trabajo ligados a cada subfunción). Cuando la organización entra en modo de fallo, aparecerán consecuencias (efectos) que acabarán en condiciones inseguras para los actores del proceso (asuntos de panel $\mathrm{S}$ ), en defectos en el producto (asuntos de panel Q), en demoras que tienen impacto en mantener la organización con el ritmo de producción adecuado (asuntos de panel D), en averías en los medios productivos (asuntos de panel C), en efectos en la moral de las personas (asuntos de panel M), y en residuos y vertidos (asuntos de panel $\mathrm{E})^{4}$. Los equipos FMEA elaboran los Diagramas Ishikawa a partir del cruce de efectos o no 
conformidad a modos de fallo y facilitan esta información a los GAP. Los GAP, aplicando observación cuidadosa del escenario y siguiendo la secuencia de descarte del protocolo de diagnóstico rápido basado en el conocimiento FMEA, y aplicando las ocho disciplinas de la resolución de problemas, devuelven en muy poco tiempo (en unos pocos minutos) la normalidad a la organización sellando el punto de escape (el lugar del proceso por donde el defecto emerge). La consecuencia del problema es que el GAP eleva el estándar a una condición más robusta (mejora). Este es el modo en el que la organización establece una dinámica que hace que la ocurrencia de los modos de fallo sea cada vez menor en los elementos de trabajo, que la detectabilidad del problema sea cada vez más efectiva y temprana, y que las no conformidades resultantes de entrar en modo de fallo tengan el menor alcance e incluso la reducción de la severidad. En segundo lugar, la organización establece otro canal de resolución de problemas orientado a incrementar el conocimiento de causas raíz (desconocidas) de los modos de fallo potenciales aplicando técnicas de investigación, como es la herramienta metodológica definir, medir, analizar, mejorar y controlar (DMAIC) para los problemas cuyas raíces están en la variabilidad de causas aún desconocidas en proceso, las cuales provocan que la organización entre en modo de fallo. Un modo de fallo progresa desde que se inicia una condición patológica en un elemento de trabajo (por fatiga, fractura, desgaste, etc.) y tras un intervalo más corto, o más largo de tiempo, acaba generando una no conformidad. Este otro tipo de resolución de problemas, en ocasiones tiene un motivo de innovación o de reducción del desperdicio basado en el análisis de la cadena de valor, de evaluación comparativa, de revisión de los seis desperdicios de LM, de hallazgos de auditorías de producto o proceso, de análisis forense (Pareto) de defectos detectados por los GAP, o de otros mecanismos que la organización tiene habilitados en su modelo de negocio como motor de la mejora continua.

\section{Resultados}

Para implantar cada una de las relaciones de comunicación y transmisión de información definidas por el VSM, la dirección despliega y hace realidad el decálogo, las políticas, las rutinas, y el conjunto de hábitos que dan forma al entramado del VSM siguiendo el paradigma LM.

El sistema de rendición de cuentas, a todos los niveles, se basa en el conjunto de las Key Performance Indicators (KPI), en el criterio de alarma de cada KPI (rendimiento actual frente al esperado), $y$ en el plan de acción derivado de las discrepancias que el sistema de alarmas hace visible. Cada uno de los cinco subsistemas definidos por el VSM ha de ser enjuiciado en base a una lista de cuestiones críticas relativas a su misión.

La forma en que se evalúa el desempeño tiene consecuencias drásticas en la orientación del esfuerzo de mejora continua (Goldratt, 2010). Por ejemplo, una organización que opera bajo el paradigma MP tomará típicamente en un centro de trabajo una KPI como "número de unidades producidas en el turno" (o, por agregación, en el día, la semana, el mes, etc. en los diversos niveles de la jerarquía). Esta es una métrica máses-mejor, que tiene consecuencias (perversas), como pagar incentivos sin que necesariamente haya habido ningún impacto favorable en la cuenta de resultados, almacenar productos que no se van a vender en una ventana larga de tiempo, generar obsoletos, dificultar el flujo FIFO por saturación de espacios de almacenaje, generar problemas de tesorería por inmovilizado, etc. Una organización que opera bajo el paradigma LM preferirá una KPI para evaluar el desempeño del proceso productivo o la adherencia al plan de fabricación como fabricar-según-programación, aplicando curvas de quemado; impulsar mejoras de cambio rápido que incrementen la flexibilidad de la organización consiguiendo mayor fluidez en el flujo y como consecuencia, menos stock; aplicar el coeficiente de rotación del inventario para reducir el montante de inmovilizado por inventarios; revisar el tiempo de activación o el tiempo de espera para reducir el tiempo de espera de las piezas, mejorar la efectividad total de los equipos que promueve la reducción sistemática de las seis grandes fuentes de desperdicio, etc.

De modo que la determinación de las KPI que llevamos al cuadro de mando en cada nivel de la organización tiene consecuencias relevantes sobre la actitud que adoptarán los agentes de la organización. Y eso pone de relieve otro de los problemas en la evaluación del desempeño de los procesos, que es el alineamiento entre los diversos niveles. Desde la perspectiva del VSM esa clase de conflictos la vemos en forma de un nivel bajo de colaboración entre unos subsistemas y otros (síndrome de los silos de los óptimos locales).

\subsection{Sistemas de Información para las unidades operacionales}

Las unidades operacionales necesitan un flujo de información para orquestar las actividades de los actores (responsable, contable, consultado, informado) de la matriz de asignación de responsabilidades (RACI) desde cada evento inicial hasta completar un hilo que produce los bienes y servicios requeridos por los clientes. Las carencias en cualquiera de las necesidades de información de las unidades operacionales conducirán a situaciones 
situaciones de MURI (agobio, erosión de la motivación, urgencias, inestabilidad, etc.), MUDA (desperdicios en la cadena de valor), o MURA (desequilibrios entre las unidades operacionales, desbalance de cargas entre operarios ligados a una actividad, lotes grandes que restan flexibilidad a las operaciones, largos tiempos para cambios de utillajes, etc.). Para resolver estos problemas, disponemos de herramientas como las 5S, las instrucciones de trabajo y la forma en que las entrenamos, la gestión visual, el sistema de alarmas, los sistemas anti-error, el mantenimiento proactivo en primer escalón, y demás ingredientes del paradigma LM que tienen como objetivo procurar la estabilidad (hacer que la organización sea predecible en su conducta y rendimiento).

El marco para evaluar las unidades operacionales es SQDCME. El subsistema de auditorías por capas demerita actos y condiciones inseguras, debilidades en orden y limpieza (5S), carencias en competencias y en flexibilidad de los operarios frente a los requerimientos del estándar, debilidades en la gestión visual, carencias y/o riesgos en los protocolos de gestión de excepciones y anomalías, y en general, revela la madurez con la que se están gestionando los riesgos en las operaciones y cuidando la satisfacción de los clientes externos e internos. La efectividad total de los equipos, con el análisis de las seis grandes pérdidas de Nakajima(1991), es una de las KPI más relevantes para evaluar las unidades operacionales. Hace visible el aprovechamiento de la capacidad disponible y las variaciones del rendimiento en velocidad y en calidad. Es un indicador sensible a la eficacia de los cambios de utensilios, sensible a las averías, a las microparadas, y a la calidad. Es conveniente revisar las necesidades de información de las unidades operacionales en base al modelo "Tuberías y Charcos". Para ello, es necesario distinguir entre tres tipos diferentes de tuberías en cada uno de los procesos MOP, COP y SOP, ya comentados:

- Tubería higiénica: encargada de la función de proceso previsto. Implementa también la gestión de las excepciones (sistema de control visual). Cuanto más efectivo es esta gestión de las excepciones, menos trabajo reactivo sucede en la organización.

- Tubería reactiva: encargada de reaccionar a los problemas de la tubería higiénica.

- Tubería de mejora continua: encargada de aplicar la gestión del cambio para elevar el rendimiento de la tubería higiénica.

Lo descrito hasta el momento en referencia a las unidades operacionales, corresponde a la tubería higiénica (Figura 4), que es la tubería que genera valor de negocio (por ejemplo, el proceso de soldadura, de ensamblaje, de facturación, de compras, de entradas, etc.).
Figura 4 Tubería higiénica (elaboración personal)

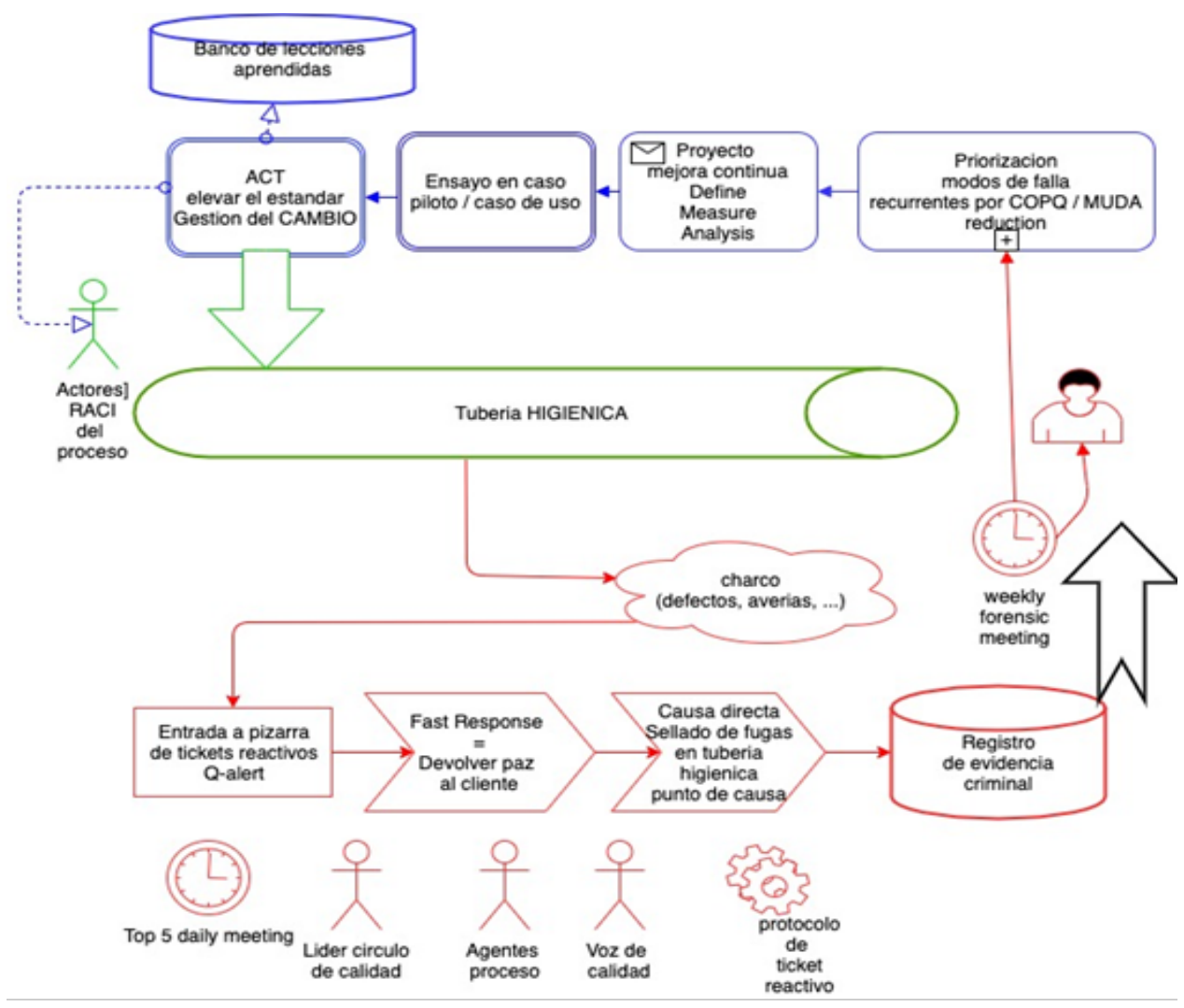


En este sentido, las necesidades de información de todo proceso de una organización se pueden articular con historias de usuario para cada tipo de tubería. Se muestran a continuación algunas historias de usuario a modo de ejemplo para cada uno de los tipos de tubería.

\subsubsection{Historias de usuario de la tubería higiénica}

Como actor con role que ejecuta tareas y controles en el proceso de ensamblado, para producir con el ritmo de producción requerido, adherido al plan de fabricación (y con ello, tener conforme el indicador fabricarsegún-programación), es necesario recibir la orden de fabricación siguiente cada vez que sale un nuevo código de producto.

Como role líder de equipo de una estación de trabajo de ensamblado, para aplicar gestión visual a las condiciones de excepción que pueden darse en la organización, es necesario que las personas del GAP tengan a la vista el sistema de control visual vigente en cada momento. Cuando el sistema de control visual está en color verde (ninguna excepción en curso) basta la señal verde; cuando está en color amarillo, es necesario que se haga visible el instante en el que se ha levantado la excepción, cuál es la excepción en concreto y el momento más tardío aceptable para haber regresado a la condición verde; finalmente, cuando el sistema de control visual está en color rojo y requiere escalamiento, es necesario que además se haga visible en qué canal se encuentra dicho escalamiento así como la realimentación actualizada del canal de soporte que está atendiendo dicho escalamiento.

Como actor con role de jefe que rinde cuentas de una sección de ensamblado para que las personas a su cargo y él mismo tengan una gestión visual sobre lo que está sucediendo en el turno, es necesario una monitorización en tiempo real de la curva de quemado del plan de fabricación.

\subsubsection{Historias de usuario de la tubería reactiva}

Como miembro del GAP de una estación de cardas, para tener un seguimiento efectivo de las no conformidades vigentes, es necesario ver los tickets reactivos en una pizarra SCRUM. Además, es necesario ver en cada ticket la no conformidad, su nivel de severidad, su alcance, la fecha-hora en la que ha ocurrido, dónde ha sido detectada, qué agente lo ha detectado, la categoría del ticket (tipo de problema), qué estado actualmente tiene el ticket y cuándo ha sido el último cambio de estado del ticket. Puesto que lo normal es que no haya ningún ticket reactivo vivo y ocasionalmente uno (rara vez más de uno), no es necesario ningún sistema de priorización.
Como intendente del proceso de cardas, para evaluar el desempeño del tratamiento de no conformidades en un proceso, es necesario que semanalmente cada sección de cardas tenga su informe de evaluación de desempeño, donde se puede considerar la siguiente información relevante:

- Tasa de salida vs tasa de entrada para verificar que la organización tiene la capacidad adecuada.

- Tiempo de llegada de los tickets reactivos al cierre de semana y trayectoria temporal de las últimas 10 semanas de este indicador.

- $\quad$ Tiempo de espera de los tickets cerrados en la semana y trayectoria temporal de las últimas 10 semanas para saber lo que se tarda en cerrar los tickets reactivos.

\subsubsection{Historias de usuario de la tubería de mejora continua}

Como miembro del GAP de una estación de cardas, para tener una perspectiva acerca de cómo se aplica la mejora, necesitamos tener una gestión visual sobre el flujo de tickets de mejora continua.

Como supervisor de una estación de cardas, para que los agentes de la organización tengan una comprensión sobre el valor que genera el caudal de tickets de mejora, necesitamos que cada mes se haga visible un gráfico de cascada, donde se visualice el impacto de los tickets que han sido cerrados en el mes sobre las KPI del cuadro de mando.

\subsection{Sistemas de Información para el subsistema de coordinación}

El subsistema de coordinación lo forma el sistema de señales que actúan de disparadores para los actores. Los actores necesitan gobernar el ritmo de producción (por ejemplo, con el sistema visual de curvas de quemado), necesitan aplicar la regla parar-para-arreglar cuando sucede una anomalía en algún punto de la cadena de valor (por ejemplo, mediante el sistema de alarmas), lo que incluye los casos de escalamiento de problemas y, necesitan gestionar los tiempos de espera hasta que se cumplen las condiciones para actuar en cada paso del proceso (tareas frecuenciales, etc.).

Algunos indicadores típicos y destacables del subsistema de coordinación son: fabricar-según-programación, tiempo entre fallos, tiempo hasta reparación, tasa de situaciones de alarma (sistema de gestión visual) levantadas por cubo de tiempo, etc. 
Un aspecto fundamental en cuanto al subsistema de coordinación son los flujogramas Business Process Model and Notation (BPMN), donde es necesario hacer visible (Figura 5):

- En la calle superior encontramos los actores del proceso examinado (análisis forense semanal de tickets reactivos cuya repetición preocupa a los gestores de la organización).
- En la calle inferior encontramos los medios y conexiones que este proceso requiere (la parte más relevante de la orquestación, como registros documentados, llamadas a otros procesos, etc.).

- En la calle central encontramos el hilo de trabajo desde un evento que dispara una instancia, la secuencia de operaciones, controles, bifurcaciones, confluencias y esperas, hasta que el hilo termina su función.

Figura 5 Business Process Model and Notation (BPMN) (elaboración personal)

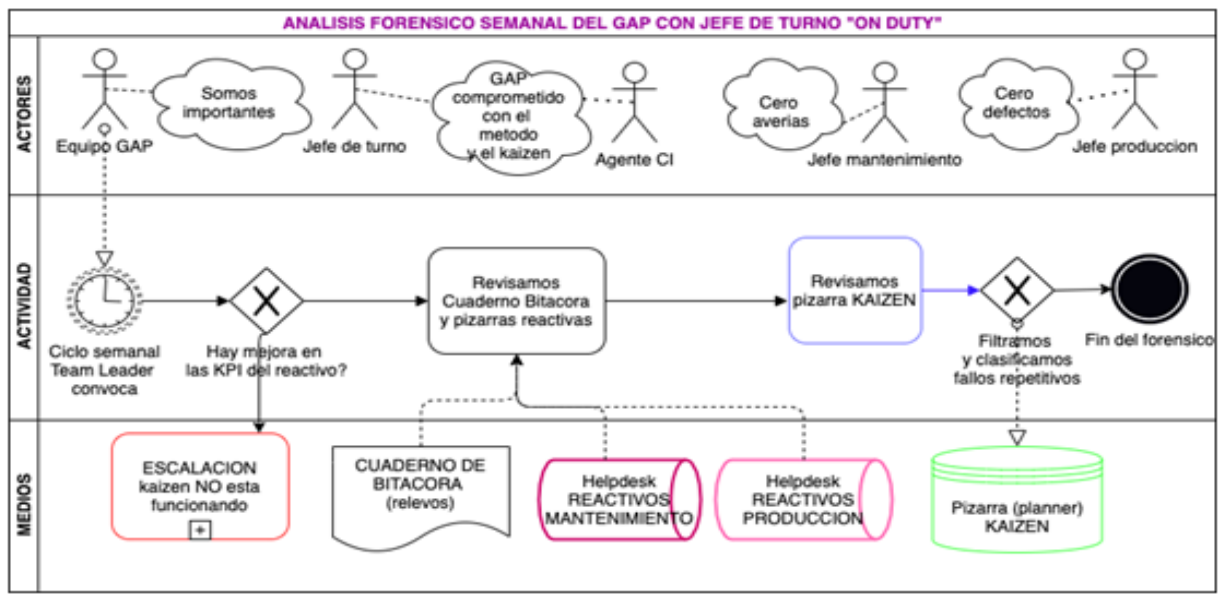

\subsection{Sistemas de Información para el subsistema de integración y sinergia}

Los sistemas de información propios de las unidades operacionales y del subsistema de coordinación están situados en su lugar de aplicación. Tiene poco interés su registro documentado, mientras que tiene alto impacto que sean entendidos por los operarios y supervisores en tiempo real. Son fácilmente representados con diagramas de tortuga, flujogramas BPMN y las instrucciones y descripciones de trabajo de cada puesto de trabajo.

El subsistema de integración y sinergia requiere de registros documentados, interesa vertebrarlo sobre las siete herramientas básicas para la calidad, y convertir datos registrados en información sobre una distribución de una población, estratos de la misma, patrones de evolución temporal, leyes de Pareto (80-20), etc. El nivel de rendimiento de una organización lo apreciamos con un análisis de la cadena de valor y con el cuadro de mando.

Del subsistema de integración y sinergia se pueden destacar diferentes KPI: variación en el uso de materiales, variación en la eficiencia laboral, utilización de la capacidad, utilización del espacio y coste de la mala calidad externa. En definitiva, métricas que permiten a los gestores de la organización determinar hasta qué punto la organización cumple con su condición objetivo.
Un cuadro de mando convierte datos en información. Por ello, un análisis efectivo de cuadro de mando (evaluar el desempeño del proceso) utiliza alguna de las siete herramientas básicas para la calidad:

- $\quad$ Perspectiva de la evolución temporal de una KPI. Se necesita saber si el nivel de rendimiento está estancado, si el rendimiento decae, o si estamos mejorando el rendimiento. Se necesita, además el contraste de esa KPI contra el rendimiento común en el mercado, así como contra los mejores del mercado. Por ejemplo, en una revisión mensual de operaciones, se necesita ver la serie temporal de la evolución de la efectividad total de los equipos de una línea de fabricación en los últimos 24 meses y, además un gráfico de cascada que explica cada salto (cambio realizado y su impacto).

- Gráficas de Pareto. Por ejemplo, para analizar el indicador desviación en coste de materiales vs estándar en escandallo, los gestores de la organización necesitan observar con qué categorización el propietario del proceso ha conseguido, junto a su equipo, encontrar una ley 80-20 a fin de proponer un plan de acción para mejorar y que inspire confianza en cuanto a la orientación de los proyectos de mejora continua. 
- Diagramas de distribución (histogramas, diagramas de caja, gráficos de puntos, etc.). En la revisión regular de nuevos proyectos (lanzamiento de nuevos productos), por ejemplo, los gestores de la organización necesitan ver evidencias acerca de los estudios de estabilidad y capacidad a lo largo del progreso del proyecto hasta el comienzo de la producción. También necesitan ver qué causas asignables se han ido identificando y resolviendo hasta tener unas condiciones de proceso estable, base para el estándar industrial en la serie y necesitan ver cómo se han ido resolviendo los problemas de variabilidad en forma de causas verificadas y contramedidas aplicadas.

\subsection{Sistemas de Información para el subsistema de inteligencia y adaptación}

El subsistema de inteligencia y adaptación es tanto más competente cuanto mejor sabe identificar y pronosticar los cambios en el entorno de la organización, y cuanto mejor sabe dirigir el curso de los cambios (orientación del esfuerzo morfoadaptativo).

El conocimiento del entorno es vital para el despliegue estratégico. Implica ocuparse en definir estrategias de precios, la gestión del nombre y reputación de la marca, la conversión de las interacciones con los clientes en ventas futuras, la habilidad para personalizar los productos a perfiles de clientes, la gestión del ciclo de vida de los clientes, la integración de la cadena de suministro, etc. Para ello, será necesario conocer lo que ocurre en el mercado y cómo el mercado percibe a la propia organización, las tendencias de los clientes, lo que ocurrirá en la cadena de suministro, en las entidades financieras, las oportunidades de fortalecer relaciones favorables en el futuro, identificación de potenciales alianzas, maniobras potenciales de los competidores, cambios en el marco jurídico y legislativo, cambios en las tecnologías, pesquisas sobre competidores, etc. Por otra parte, el subsistema de inteligencia y adaptación se complementa con la información de lo que ocurre dentro de la organización.

Evaluar el subsistema de inteligencia y adaptación implica conocer y medir el uso que la organización hace de herramientas sobre toma de decisiones para dirigir su rumbo. Junto a ellas, también son necesarias herramientas de evaluación comparativa, herramientas para medir el nivel de cumplimiento de los objetivos a largo plazo de la organización, medir la introducción de nuevos productos en el mercado o medir la falta de acierto en conectar con las necesidades del mercado.

\subsection{Sistemas de Información para el subsiste- ma de definición de identidad y política}

Los sistemas de información del subsistema de definición de identidad y política deben permitir elaborar la identidad de la organización, su visión en cuanto a lo que quiere ser, su filosofía, sus principios, sus valores, etc. Para ello, es necesario disponer de información procedente de todos los implicados en el propio subsistema de definición de identidad y política (accionistas, empleados, clientes, proveedores, etc.) y, de manera más general, información procedente de personas o grupos del entorno que tengan o puedan tener algún tipo de relación con la organización (otras partes interesadas).

El subsistema de definición de identidad y política despliega la visión para que sea entendida por todos y sea redefinida para cada subsistema (HOSHIN KANRI). Por otra parte, comprueba la existencia de herramientas que permitan valorar si la organización está realmente cumpliendo con su misión y si es necesario modificarla. Por último, aplica herramientas para hacer efectivo el código ético, a fin de reforzar los mensajes de identidad corporativa o para capturar alertas de crisis de valores en la organización.

\subsection{Sistemas de Información en una organización manufacturera}

Los sistemas de información están en la esencia de los procesos higiénicos (lo que la organización hace para cumplir su finalidad), de los procesos reactivos (lo que la organización hace para reaccionar a no conformidades), y de los procesos de mejora continua (lo que la organización hace para seguir siendo viable en el futuro). En los procesos higiénicos, los sistemas de información se ponen en valor al orquestar a los actores para satisfacer a los clientes sin incurrir en más nivel de MUDA que el previsto en el estándar. En el resto, procesos reactivos y procesos de mejora continua, los sistemas de información se ponen en valor siendo parte del engranaje de solución de problemas y desarrollo de talento corporativo.

En la resolución de problemas, hay cuatro preguntas secuenciales críticas:

- Q1: ¿Qué desviación está pasando en el sistema?

- Q2: ¿Por qué? ¿Cuál es la causa directa de esta desviación? ¿Cuál es la causa raíz?

- Q3: ¿Qué contramedidas podemos aplicar sobre las causas? 
- Q4: ¿De qué debemos ocuparnos a continuación?

Las pretensiones de esta evaluación de los sistemas de información de una organización manufacturera empiezan por detectar qué está ocurriendo (hallazgos). Por ejemplo, imaginemos que un hallazgo es que el sistema de información encargado de extender la visión a todos los empleados (relación que une el subsistema de definición de identidad y política con el resto de subsistemas) no es eficaz en la realización de su trabajo. No podemos aún ofrecer una solución ya que no conocemos por qué no es eficaz. Por tanto, el sistema de información higiénico termina con el hallazgo, y el hallazgo es canalizado hacia el proceso de solución de problemas, uno de los cuatro pilares del paradigma LM.

El proceso de solución de problemas, en este caso pudiera ser, siguiendo con el ejemplo, que se lleve a cabo un proyecto apoyado por expertos actitudinales sobre la aceptación y la recursión de la visión de la organización. Será el contexto de este proyecto donde se investigarán las causas raíz, se ensayarán soluciones y se implantará la solución verificada.

\section{Conclusiones}

A lo largo de este trabajo se han desarrollado criterios para evaluar los sistemas de información de una organización manufacturera. Estos sistemas son parte esencial de todos los procesos en la organización. De la misma manera, se muestra cómo aplicar el ciclo planear-hacer-comprobaractuar (PDCA) para realizar los cambios necesarios en dichos sistemas como respuesta a la identificación de problemas. A fin de mantener el foco del trabajo, no se ha desarrollado la aplicación de las ocho disciplinas para la resolución de problemas, si bien en el espacio del ticket reactivo y en el de los proyectos de mejora continua, la aplicación talentosa de tales disciplinas marca una clara diferencia en agilidad y efectividad.

Por otra parte, también queremos remarcar que para llevar a cabo una auditoría certera se requiere del GENCHI GENBUTSU, es decir, ir al lugar y observar por parte del equipo auditor. Por eso, la observación cuidadosa, atenta, incisiva, y crítica en los detalles es la clave para formular un problema de forma científica.

En futuros trabajos se profundizará en el pilar de solución de problemas, sus ocho disciplinas, el ciclo PDCA en la gestión del cambio, y las dinámicas propias de los equipos de alto desempeño. Se pasará por tanto de los hallazgos a sus causas, de las auditorías a los diagnósticos, de la generación de ideas sobre contramedidas a las causas, completando la evaluación de los sistemas de información más allá de los procesos higiénicos, ofreciendo así pautas para diseñar una organización manufacturera con marcadas características de viabilidad.

\section{Referencias}

Beer S (1979) The Heart of Enterprise. John Wiley \& Sons

Beer S (1981) Brain of the Firm, 2nd ed. John Wiley \& Sons

Beer S (1985) Diagnosing the System for Organizations, John Wiley \& Sons

Cuatrecasas L (2000) Organización de la producción y dirección de operaciones. Editorial Centro de Estudios Ramón Areces

Frazelle E (2001) World-Class Warehousing \& Material Handling. McGraw-Hill

Goldratt EM (2010). La Meta. Un proceso de mejora continua. Tercera edición revisada. Ediciones Granica S.A.

Koontz H, Weihrich H (1990) Administración. 9ª Edición. McGraw-Hill

Liker JK (2004) The Toyota Way: 14 Management Principles from the World's Greatest Manufacturer. McGraw-Hill

Liker JK, Meier H (2006) The Toyota Way Fieldbook. McGraw-Hill

Nakajima S (1991) Introducción al TPM: mantenimiento productivo total. Productivity Press.

Pérez Ríos JM, Sánchez Mayoral P, Puche Regaliza JC (2008) Sistemas de información y Cibernética Organizacional. Insights on Current Organization Engineering. II International Conference on Industrial Engineering and Industrial Management - XII Congreso de Ingeniería de Organización, p. 427-437. Uni-versidad de Burgos.

Porter ME (1987) Competitive Advantage. Free Press, a Division of Macmillan, Inc.

Womack JP, Jones DT, Roos D (1990) The Machine that Changed the World. Rawson Associates 